\title{
Biomarkers of Neurodegeneration in Autoimmune-Mediated Encephalitis
}

\author{
Peter Körtvelyessy ${ }^{1,2,3,4 *}$, Harald Prüss ${ }^{3,4}$, Lorenz Thurner ${ }^{5}$, Walter Maetzler ${ }^{6,7,8}$, \\ Deborah Vittore-Welliong ${ }^{9}$, Jörg Schultze-Amberger ${ }^{10}$, Hans-Jochen Heinze ${ }^{1,2,11}$, \\ Dirk Reinhold ${ }^{12}$, Frank Leypoldt ${ }^{8}$, Stephan Schreiber ${ }^{13}$ and Daniel Bittner ${ }^{1,2}$
}

\begin{abstract}
${ }^{1}$ Department of Neurology, University Hospital Magdeburg, Magdeburg, Germany, ${ }^{2}$ German Center for Neurodegenerative Diseases Magdeburg, Magdeburg, Germany, ${ }^{3}$ Department of Neurology, Charité-Universitätsmedizin Berlin, Berlin, Germany, ${ }^{4}$ German Center for Neurodegenerative Diseases Berlin, Berlin, Germany, ${ }^{5}$ José Carreras Center for Immuno- and Gene Therapy and Internal Medicine I, Saarland University Medical School, Homburg, Germany, ${ }^{6}$ Department of Neurodegeneration, Hertie Institute for Clinical Brain Research (HIH), University of Tübingen, Tübingen, Germany, ${ }^{7}$ German Center for Neurodegenerative Diseases Tübingen, Tübingen, Germany, ${ }^{8}$ Department of Neurology, University Hospital Schleswig-Holstein, Kiel, Germany, ${ }^{9}$ Department of Neurology and Epileptology, Universitätsklinikum Tübingen, Universität Tübingen, Tübingen, Germany, ${ }^{10}$ Department of Neurology, Median Clinic Kladow, Kladow, Germany, ${ }^{11}$ Department of Behavioral Neurology, Leibniz Institute for Neurobiology, Magdeburg, Germany, ${ }^{12}$ Department of Immunohistopathology, Institute of Molecular and Clinical Immunology, Magdeburg, Germany, ${ }^{13}$ Asklepios Department of Neurology, Brandenburg a.d. Havel, Germany
\end{abstract}

\section{OPEN ACCESS}

Progranulin (PGRN), Total-Tau (t-tau), and Neurofilament light chain (NfL) are well known Edited by: Stefan Bittner, Johannes Gutenberg-Universität Mainz, Germany

Reviewed by:

Nico Melzer,

Universität Münster, Germany Michael R. Pranzatelli, National Pediatric Neuroinflammation

Organization, Inc., United States

*Correspondence:

Peter Körtvelyessy peter.koertvelyessy@med.ovgu.de

Specialty section:

This article was submitted to Multiple Sclerosis and

Neuroimmunology, a section of the journal Frontiers in Neurology

Received: 12 May 2018

Accepted: 25 July 2018 Published: 19 September 2018

Citation: Körtvelyessy P, Prüss $H$, Thurner $L$,

Maetzler $W$, Vittore-Welliong $D$, Schultze-Amberger J, Heinze H-J, Reinhold D, Leypoldt F, Schreiber S and Bittner D (2018) Biomarkers of

Neurodegeneration in Autoimmune-Mediated Encephalitis.

Front. Neurol. 9:668.

doi: 10.3389/fneur.2018.00668 biomarkers of neurodegeneration. The objective of the present study was to investigate whether these parameters represent also biomarkers in autoimmune-mediated Encephalitis (AE) and may give us insights into the pathomechanisms of AE. We retrospectively examined the concentration of PGRN in the cerebrospinal fluid (CSF) and serum of 38 patients suffering from $A E$ in acute phase and/or under treatment. This AE cohort comprises patients with autoantibodies against: $\operatorname{NMDAR}(n=18$ patients), Caspr2 $(n=8)$, Lgi-1 $(n=10), \operatorname{GABAB}(\mathrm{R})(n=1)$, and AMPAR $(n=1)$. Additionally, the concentrations of NfL $(n=25)$ and t-tau $(n=13)$ in CSF were measured when possible. Follow up data including MRI were available in 13 patients. Several age-matched cohorts with neurological diseases besides neuroinflammation or neurodegeneration served as control groups. We observed that PGRN was significantly elevated in the CSF of patients with NMDAR-AE in the acute phase, but normalized at follow up under treatment $(p<0.01)$. In the CSF of other patients with AE PGRN was in the range of the CSF levels of control groups. T-tau was highly elevated in the CSF of patients with temporal FLAIR-signal in the MRI and in patients developing a hippocampal sclerosis. NfL was exceptionally high initially in Patients with AE with a paraneoplastic or parainfectious cause and also normalized under treatment. The normalizations of all biomarkers were mirrored in an improvement on the modified Rankin scale. The data suggest that the concentration of PGRN in CSF might be a biomarker for acute NMDAR-AE. Pathological high t-tau levels may indicate a risk for hippocampal sclerosis. The biomarker properties of NfL remain unclear since the levels decrease under treatment, but it could not predict severity of disease in this small cohort. According to our results, we recommend to measure in clinical practice PGRN and t-tau in the CSF of patients with AE.

Keywords: progranulin, neurofilament light chain, NMDAR encephalitis, Lgi-1 encephalitis, Caspr2 encephalitis, tau, autoimmune encephalitis 


\section{INTRODUCTION}

Since the appearance of antibody-mediated autoimmune encephalopathy (AE) numerous antibodies (ab) have been linked to different clinical symptoms such as limbic encephalitis, faciobrachial dystonic seizures or dementia-like symptoms (1-3). Biomarkers of neurodegeneration mirror certain pathomechanisms of neuronal or axonal loss. The measurement of these biomarkers should bear the potential to provide useful information in everyday clinical life, e.g., to monitor the immunosuppressive therapy in Patients with AE. CSF antibody titres in e.g., contactin-associated-protein-receptor-2 (Caspr2)$\mathrm{AE}$ or Leucin-rich glioma inactivated ptotein-1 (Lgi-1)-AE do not mirror the disease course in a linear way $(4,5)$. Furthermore, the clinical course in several patients suggests that an antibody titer independent pathomechanism might take place (6-8). The underlying mechanisms causing this dichotomy of clinical symptoms and antibody titer are largely unknown (8). One possible explanation could be the effect of the long survival of plasma cells in the brain (9). The brain-resident plasma cells itself cannot be measured as yet, but the damage possibly caused by autoantibodies should be detected via biomarkers for neuronal and axonal loss such as t-tau, PGRN, and NfL.

Recently, a direct connection between neurodegenerative mechanisms and $\mathrm{AE}$ has been detected in AEs mediated by IgLON5 causing an atypical tauopathy (10). Vice versa a correlation between autoimmune diseases and Tar DNA-binding Protein 43 (TDP-43) mediated neurodegeneration in FTD patients has been reported (11). There is also some debate about IgA-NMDAR-Abs and IgM-NMDAR-Abs $(3,12,13)$ causing dementia-like symptoms and mimicking neurodegenerative diseases. Histopathological examinations in patients with $\mathrm{AE}$ have been focused on the immunological mechanisms triggered and maintained by the antibodies (8) disregarding a systematic research for markers of neurodegeneration so far. Only one case report of a Lgi-1 antibody positive patient presenting some neurodegenerative markers has been reported at autopsy, without witnessing pathological changes in alpha-synuclein, beta-amyloid, or neurofibrillary tangle (14). MRI findings and long-term neuropsychological data also suggest an involvement of the frontal and temporal lobes in the clinical course of the NMDAR-AE and voltage-gated-potassium channel (VGKC)complex-mediated AE (15-18). Another group has looked at the glial fibrillary acid, NfL and t-tau levels in patients with suspected AE (19). Their group only encompassed four patients with NMDAR-AE and one Lgi-1 patient with most of them having a status epilepticus (SE) before, as SE is known for confounding the protein levels in the CSF $(20,21)$. They found higher NfL

\footnotetext{
Abbreviations: Ab, Antibody; AD, Alzheimer's disease; AE, Autoimmune mediated Encephalitis; AMPAR, alpha-amino-3-hydroxy-5-methyl-4isoxazolepropionic acid receptor; Caspr2, Contactin associated protein 2; CSF, cerebrospinal fluid; FTD, Frontotemporal dementia; $\operatorname{GABAB}(\mathrm{R})$, Gabaaminobutyrat-B subunit receptor; Lgil, Leucin-rich glioma inactivated protein1; NMDAR, N-methyl-D-aspartate receptor; NfL, Neurofilament light chain; PGRN, Progranulin; SE, Status Epilepticus; t-tau, T-tau; VGKC, Voltage-gated potassium channel.
}

and t-tau levels in all patients, which is most likely due to the SE before. In pediatric opsoclonus-myoclonus syndrome caused by antibodies with intracellular epitopes, immunosuppressive treatment has shown to decrease the CSF-Neurofilament light chain levels together with a concomitant clinical improvement $(22,23)$.

Here, we examined concentrations of PGRN, NfL, and ttau, well-established biomarkers of neurodegeneration, in CSF and serum of 38 patients with antibody positive AE. The aim of this study is to investigate if these proteins are possible biomarkers in Patients with AE. Also, the knowledge about the biomarkers of neurodegeneration CSF-levels may give clues about the pathological mechanisms in these patients.

\section{METHODS \\ Clinical Cohort}

This retrospective study was performed according to the local ethical committees in Berlin, Potsdam, Brandenburg, Magdeburg and Bielefeld, respectively. All patients gave written and informed consent (ethics committee approval number 100/16). We included only patients with a proven AE by clinical symptoms as recommended by Graus et al. (24) and detection of pathological antibodies with extracellular epitopes via indirect immunofluorescence tests. The samples of AE-Patients were collected from April 2013 until October 2017 in Berlin, Potsdam, Tübingen, Bielefeld, and Magdeburg where their samples were initially stored at $-80^{\circ} \mathrm{C}$ and sent to Magdeburg. Every sample was stored in Magdeburg at $-80^{\circ} \mathrm{C}$ and all biomarkers were measured in Magdeburg. All samples were run in duplicate with the mean taken as result. Samples were measured over time and not in a batch.

All patients received a lumbar puncture as part of the diagnostical work up when presenting for the first time on the ward and for antibody titre control in follow up depending on each individual disease course. At least $5 \mathrm{ml}$ up to $13 \mathrm{ml}$ CSF was taken and serum was collected in serum separator tubes and centrifuged at site. Every patient $(n=38)$ suffered from a limbic encephalitis including its variants, e.g., limbic encephalitis together with vegetative and/or peripheral neurological symptoms. All patients improved under immunosuppressive therapy. Treatment comprised methylprednisolone (dosage ranges from $3 \mathrm{~g}$ up to $18 \mathrm{~g}$ during disease course), cyclophosphamid or rituximab (with a minimum dosage of 2g), plasmapheresis or ivIG. We had no patients with a relapse in this cohort. None of the patients had a status epilepticus before lumbar puncture confounding the biomarker levels because of neuronal and axonal death due to the status epilepticus.

Two patients with other antibodies targeting extracellular epitopes [AMPAR and $\operatorname{GABAB}(\mathrm{R})$ ] were not considered in the statistical analysis but for Figure 3 to illustrate the biomarker and MRI timeline an AE patients. See Table 1 for the different $\mathrm{AE}$ cohorts used per biomarker. We had follow up data in 13 patients. All other patients (samples) were categorized into either initial/acute phase or under treatment. 
TABLE 1 | Epidemiological data.

\begin{tabular}{|c|c|c|c|}
\hline Biomarker & Antibody & Initial [age/sex] & Follow up [age/sex] \\
\hline \multirow[t]{3}{*}{ Progranulin } & NMDAR & 6 [28.9/6:0] & $17[27.1 / 16: 1]$ \\
\hline & Caspr2 & 8 [61.9/2:6] & 5 [67.1/0:5] \\
\hline & Lgi-1 & 7 [69.0/5:2] & 6 [63.2/3:3] \\
\hline \multirow[t]{3}{*}{ T-Tau } & NMDAR & 3 (38.7/3:0] & 3 (38.7/3:0] \\
\hline & Caspr2 & 5 [69.6/0:5] & 5 [69.6/0:5] \\
\hline & Lgi-1 & 3 [63.7/2:1] & 3 [63.7/2:1] \\
\hline $\mathrm{Nfl}$ & NMDAR & $3(38.7 / 3: 0]^{\star}$ & 13 (30.2/12:0] \\
\hline \multirow{2}{*}{ VGKC-group \{} & Caspr2 & $6(68.3 / 1: 5]$ & 5 [67.1/0:5] \\
\hline & Lgi-1 & 3 [63.7/2:1] & 3 [63.7/2:1] \\
\hline Control group young & None & 24 [29.9/18:6] & * \\
\hline Control group old & None & 21 [60.0/9:12] & * \\
\hline
\end{tabular}

Overview of the single groups tested in this study. Number of patients included in bold, sex and mean age are listed.

\section{Magdeburg Group}

Patients with AE who were identified and treated in Magdeburg $(n=13)$ build a special cohort, because we could e.g., compare serial MRIs to look for AE caused lesions, basic CSF parameters, the outcome with the modified Rankin scale, $t$-tau, and other biomarker levels in the CSF and ab titer. The modified Rankin scale in this cohort was assessed by two experienced neurologist (PK, DB). Six out of thirteen had a paraneoplastic origin and one patient a postinfectious origin of the AE.

\section{CSF-Neurofilament Light Chain Measurements}

We divided the AE cohort into three groups regarding the CSFNfL measurements in order to be able to perform a sufficient statistical analysis (see Table 1). One group with voltage-gated potassium channels (VGKC) mediated AE (comprising the Caspr2 and Lgi-1 patients) subdivided in an "initial" $(n=9)$ and "under treatment," meaning after several immunosuppressive therapies, subgroup $(n=8)$ and one group with NMDA patients under treatment $(n=13)$ (see Table 1 and Figure 1). There were not enough NMDA patients who would fit into an initial/acute phase group $(n=3)$. Therefore, this group could unfortunately not be part of the statistical analysis.

Neurofilament light chain was measured with a commercial ELISA (Umandiagnostics, Sweden, catalogue number 10-7001 $\mathrm{CE})$. The sensitivity of this assay is $31 \mathrm{pg} / \mathrm{ml}$. The cut-off for pathological levels was set at $3523 \mathrm{ng} / \mathrm{ml}$ (mean $(1823[\mathrm{ng} / \mathrm{ml}])+$ 2 standard deviation $(850[\mathrm{ng} / \mathrm{ml}])$ above). Intraessay coefficient of variance is $7.4 \%$ and interessay coefficient of variance is $6 \%$. NfL is a stable protein, which can be measured in the CSF even though the sample was on room temperature for up to 8 days (25). Therefore, we could measure NfL in samples not collected at Magdeburg, We used an already established control group at Magdeburg $(n=34$, mean age $=64.4$, CSF$\mathrm{NfL}=1823 \pm 850[\mathrm{ng} / \mathrm{ml}])$ comprising patients with other than neuroinflammatory or neurodegenerative diseases (e.g., headache, suspicion of infection in the CNS etc.).

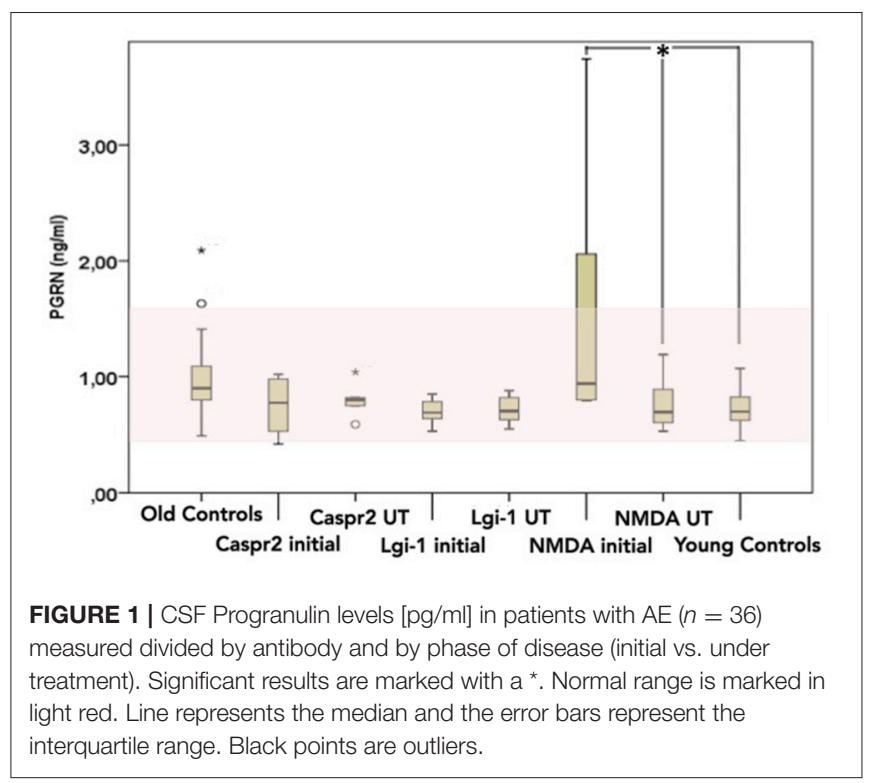

\section{Total-Tau Measurements}

The correct measurement of t-tau due to manufacturer's instructions requires different than standard processing of the CSF samples to create a cell-free sample excluding this parameter from a retrospective study. We yielded t-tau levels only in the Magdeburg group, as t-tau is part of the routine in Magdeburg but not in the other centers involved. Total-Tau levels were determined using a commercially available singleparameter ELISA kit [Innogenetics, Ghent, Belgium, catalogue numbers: 81572 (962-CE) and 81573] established in our routine diagnostical work up. Intraessay coefficient of variance is $13.2 \%$ and interessay coefficient of variance is $11.5 \%$. The pathological levels were considered according to the manufacture guidelines.

\section{CSF-Progranulin Measurements}

We measured PGRN in CSF and serum of 36 Patients with AE. We divided our AE cohort $(n=36)$ into three groups according to the antibody causing the limbic encephalitis when looking statistically at the CSF-PGRN levels: one Lgi-1 group ( $n=10$, mean age $=69.2)$, one Caspr2 group $(n=8$, mean age $=61.9)$ and a NMDAR group $(n=18$, mean age $=27.1)$. These three groups were subdivided into two subgroups respectively one before and one after initiating immunosuppressive therapy (again, called "initial" or "under treatment") (see Table $\mathbf{1}$ and Figure 2).

A commercial ELISA was performed to determine the levels of PGRN (Human Progranulin ELISA Kit, Mediagnost, Reutlingen, Germany, catalogue number E103) according to the manufacturer's instructions. Intraessay coefficient of variance is $4.4 \%$ and interessay coefficient of variance is $8.0 \%$.

We established two control groups for PGRN measurements. Since PRGN levels are age dependent, we build a younger control group ( $n=24$; mean age 29.3 years; 18-40yrs) and one older group $(n=39$; mean age 66.3 years, 


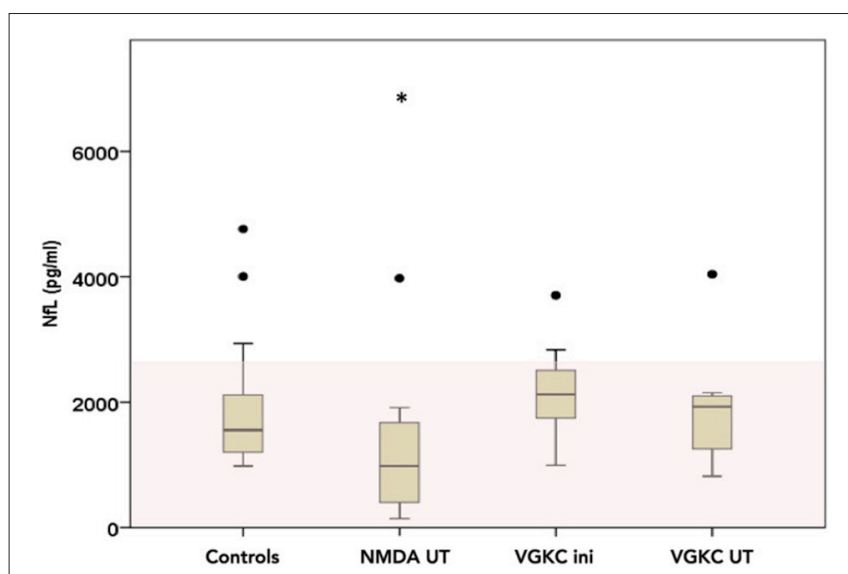

FIGURE 2 | CSF-Neurofilament light chain (NfL) levels divided into three possible groups (NMDA under treatment $n=14$, VGKC initial $n=7$ and under treatment $n=6$ ) as box plots (number of measurements, $n=30$ ). The area in light red marks the normal range. Please note that all levels are inside the normal range. Line represents the median and the error bars represent the interquartile range. White points mean outlier and star extreme outliers.

50-75 years) and correlated it to age. The patients from these control groups had other neurological diseases than neuroinflammatory or neurodegenerative (e.g., acute headache, excluding neuroinflammatory diseases, no epilepsy patients). CSF-PGRN level was considered pathological, when the CSFPGRN levels per control group were two standard deviation above or below the mean for each control group respectively.

\section{Antibody Detection}

Antibody detection was either performed at the antibody laboratory Bielefeld, at the University Hospital SchleswigHolstein, Department of Neuroimmunology Kiel and Lübeck or at the Institute of Molecular and Clinical Immunology Magdeburg. Standard indirect immunofluorescence tests were performed on antigen-specific transfected Hek293 cells as commercially available and used in clinical routine (EUROIMMUN, Lübeck, Germany, catalogue numbers: FA 112d-1003-6, FA 112d-1003-51, FA 1430-1003-1) for each patient revealing the specific antibody and the titer in CSF and serum.

\section{Statistics}

Statistics were calculated by SPSS 21.0 (IBM, Seattle, USA). Since group size and variances were not equal non-parametric tests were applied. For comparison of more than two different groups Kruskal-Wallis test was used with tamhanes post-hoc analysis. Group comparison of two groups was analyzed with Mann-Whitney-U test when they were independent and with the Wilcoxon test when the samples were paired to each other. For correlational analysis of serum and CSF PGRN Spearmann-rho correlation was applied. Tests were considered significant when reaching $p<0.05$. There was no test for outliers applied.

\section{RESULTS \\ Cohorts}

Since the CSF-PGRN level is age-dependent we established a younger control group aged $18-40$ years $(n=24$, CSFPGRN $=0.72 \pm 0.17 \mathrm{ng} / \mathrm{ml}$, Serum-PGRN $=36.4 \mathrm{ng} / \mathrm{ml}$, mean age $=29.3$ years $)$ and a control group $50-75$ years $(n=39$, CSFPGRN $=0.94 \pm 0.22 \mathrm{ng} / \mathrm{ml}$, Serum-PGRN $=28.5 \mathrm{ng} / \mathrm{ml}$ mean age $=66.3 \pm 9.8$ years). Spearman correlation statistics revealed a significant correlation between CSF-PGRN and age $(r=0.275$, $p=0.02$ ).

In the Magdeburg group 9/13 had a FLAIR-intense signal in the limbic system on the MRI. Five out of thirteen patients developed a hippocampal sclerosis due to AE (see Table 2 and Figure 3). Every patient with pathologically elevated t-tau levels developed a hippocampal sclerosis. The one patient, who developed a hippocampal sclerosis without elevated t-tau levels but elevated NfL concentrations, was administered after he was already treated and had a hippocampal sclerosis. Therefore the CSF was taken and measured $\sim 8$ months after beginning of the $\mathrm{AE}$ and treatment. On the contrary only 4 out of 7 patients with pathological NfL levels developed a hippocampal sclerosis. Every marker of neurodegeneration and the modified Rankin scale (mRS) decreased after initializing the immunosuppressive treatment paralleled by a decrease in antibody titre (see Figure 3 for examples and Table 2 for the follow up data).

\section{Neurofilament Light Chain}

$\mathrm{NfL}$ was pathologically high $(>3523 \mathrm{pg} / \mathrm{ml})$ in $7 / 23$ patients at different stages of the AE (see Figures 2, 3 and Table 2). Out of these seven patients, four had a paraneoplastic origin of the $\mathrm{AE}$ and one a postinfectious origin. Furthermore, 5/7 patients had a FLAIR-intense signal in the limbic areas on the MRI, which normalized during immunosuppressive treatment. This decrease in FLAIR signal was mirrored by a decrease in NfLlevels reaching normal NfL levels during disease course (see Figure 3 and Table 2). Three out of five patients who developed hippocampal sclerosis had elevated CSF-NfL levels additionally to the also elevated t-tau. Solely elevated CSF-NfL was found in 4 patients. We correlated the leukocyte count to the NFL levels in the Magdeburg cohort and found no correlation (Spearmans $r=0.625$ ).

There was a trend toward a lower NfL in the NMDA under treatment group (CSF-NfL $=1455$ [pg/ml], range 142$6841[\mathrm{pg} / \mathrm{ml}])$ compared to the VGKC under treatment group $($ CSF-NfL $=2164[\mathrm{pg} / \mathrm{ml}]$, range 821-4039 [pg/ml]) (MannWhitney $\mathrm{U}$ test $p=0.052 \mathrm{Z}=-1.941)$, while there was no difference comparing the VGKC subgroups initial vs. under treatment (Wilcoxon test $p=0.735$ and $\mathrm{Z}=-0.338$ ).

\section{Total Tau}

Looking at the initial t-tau in our patients (before initiating the treatment) revealed a pathologically high t-tau in 4 patients (see Table 2). All 4 patients had MRI-FLAIR intense signals in the temporal lobe/limbic system and subsequently a hippocampal sclerosis. Immunosuppressive treatment did show an effect on 
TABLE 2 | Biomarkers in the Magdeburg cohort.

\begin{tabular}{|c|c|c|c|c|c|c|c|c|c|c|c|}
\hline & Antibody & Titer & Age & Year & T-tau & NFlight & CSF-PGRN & Serum-PGRN & Cell count & mRS & Comment \\
\hline & & \multirow[t]{2}{*}{ CSF/Serum } & Onset & & $\mathrm{pg} / \mathrm{ml}$ & $\mathrm{ng} / \mathrm{ml}$ & $\mathrm{pg} / \mathrm{ml}$ & $\mathrm{pg} / \mathrm{ml}$ & CSF & & \\
\hline & & & & & $>370$ & $>3488$ & $<0.54-1.4>$ & $<18-54>$ & $<4$ cells $/ \mathrm{mm} 2$ & & \\
\hline \multirow{2}{*}{ Patient 1} & & none/1:375 & & 2015 & 133 & 2152 & 0.8 & 32.5 & 0 & 3 & Paraneoplastic origin \\
\hline & & none /1:128 & & 2015 & 108 & 2055 & 0.61 & 30.62 & 1 & 3 & Hippocampal sclerosis \\
\hline & & 1:6000/1:96000 & & 2015 & 213 & 2123 & 0.87 & 30.63 & 17 & 1 & \\
\hline \multirow[t]{2}{*}{ Patient 3} & Caspr2 & $1: 320 / 1: 3200$ & $70-75$ & $2015 \#$ & 367 & 4536 & 0.84 & 21.94 & 0 & 1 & Normal MRI \\
\hline & & 1:320/1:3200 & & $2015 \#$ & 328 & 3580 & 0.74 & 22.05 & 3 & 1 & \\
\hline Patient 4 & Caspr2 & 1:3200/1:1000 & $70-75$ & 2016 \# & 314 & 3705 & 0.95 & 35.18 & 6 & 5 & Temporal FLAIR-intense signal \\
\hline & & $1: 8 / 1: 1000$ & & 2017 & 375 & 2586 & 0.82 & 43.58 & 2 & 0 & \\
\hline \multirow[t]{2}{*}{ Patient 6} & Lgi-1 & none/1:100 & $60-65$ & $2015 \#$ & 796 & 2128 & 0.63 & 32.97 & 1 & 3 & Temporal FLAIR-intense signal \\
\hline & & none/1:32 & & 2015 & $>11$ & 2493 & 0.54 & 38.92 & 0 & 0 & Hippocampal sclerosis \\
\hline \multirow[t]{2}{*}{ Patient 7} & $\mathrm{Lg}-1$ & $1: 2 / 1: 1000$ & $65-70$ & 2017 \# & 128 & 993 & 0.74 & 19.97 & 1 & 3 & Paraneoplastic origin \\
\hline & & none/1:320 & & 2017 & 149 & 1273 & 0.73 & 23 & 0 & 0 & \\
\hline \multirow[t]{2}{*}{ Patient 8} & Lgi-1 & $1: 2 / 1: 160$ & $60-65$ & 2017 \# & 197 & 1582 & 0.65 & 27.33 & 0 & 3 & Temporal FLAIR-intense signal \\
\hline & & $1: 20 / 1: 10$ & & 2017 & 161 & 1233 & 0.68 & 30.12 & 2 & 0 & \\
\hline Patient 9 & NMDA & $1: 32 / 1: 320$ & $25-30$ & $2010 \#$ & 141 & n.a. & 0.76 & 29.11 & 1 & 5 & Paraneoplastic origin \\
\hline \multirow[t]{3}{*}{ Patient 11} & NMDA & none/1:10 & $60-65$ & $2014 \#$ & 801 & 28791 & 1.53 & 35.51 & 96 & 4 & Hippocampal sclerosis \\
\hline & & none/1:10 & & 2014 & 372 & 42286 & 1.19 & 32.9 & 43 & 0 & Temporal FLAIR-intense signal \\
\hline & & none/none & & 2015 & 192 & 3975 & 0.96 & 35.4 & 9 & 0 & Paraneoplastic \\
\hline \multirow[t]{3}{*}{ Patient 12} & GABA(B)R & $1: 320 / 1: 32$ & $50-55$ & $2016 \#$ & 135 & 32029 & 1.79 & 37.21 & 47 & 5 & Paraneoplastic origin \\
\hline & & $1: 320 / 1: 1$ & & 2016 & 168 & 21439 & 1.03 & 37.5 & 1 & 3 & Temporal FLAIR-intense signal \\
\hline & & none/1:10 & & 2016 & 126 & 3581 & 0.95 & n.a. & 1 & 2 & \\
\hline \multirow[t]{3}{*}{ Patient 13} & AMPA & $1: 32 / 1: 3200$ & $70-75$ & $2014 \#$ & 1950 & 32151 & 2.47 & 34.21 & 43 & 5 & Paraneoplastic origin \\
\hline & & $1: 8 / 1: 375$ & & 2014 & 1984 & 20354 & 1.23 & 38.22 & 90 & 4 & Temporal FLAIR-intense signal \\
\hline & & $1: 1 /$ none & & 2015 & 173 & 2892 & 0.7 & n.a. & 3 & 2 & Hippocampal sclerosis \\
\hline
\end{tabular}

Complete list of all 13 patients in the cohort where follow up data is available including antibody, antibody serum titer, age, year at which the sample has been obtained, Neurofilament light chain (NfL), Total-tau (T-Tau), CSF-Progranulin (PGRN), Serum-Progranulin, cell count, modified Rankin scale (mRS) and comments on MRI abnormalities and putative pathogenesis; n.a., not available; \#, timepoint before start of the immunosuppression. 


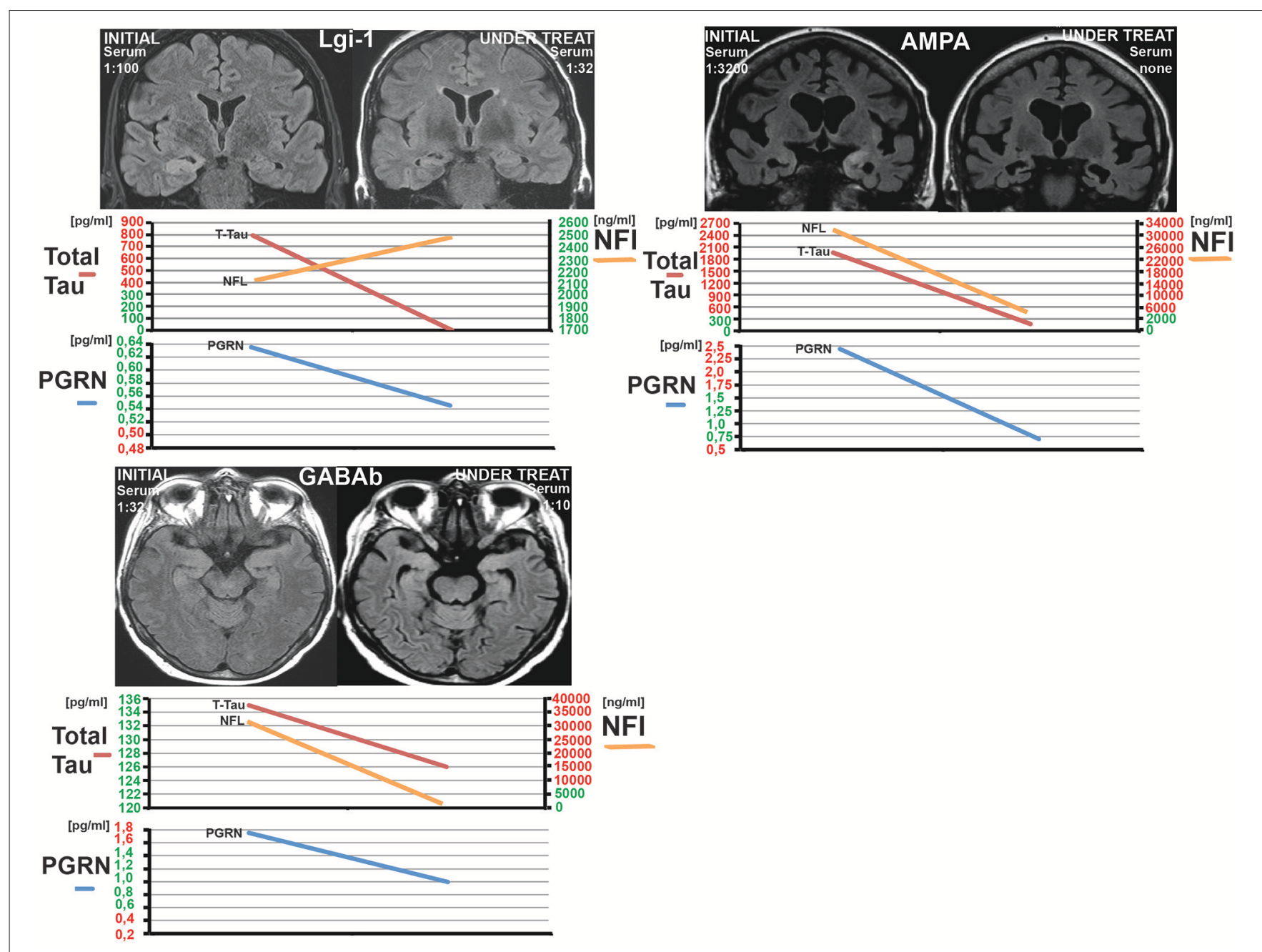

FIGURE 3 | Three examples of patients from the Magdeburg cohort (patient 5, 12, and 13 see Table 1) showing the under treatment MRI together with the CSF-Neurofilament light chain (NfL), CSF-Progranulin (PGRN), and Total tau. Normal levels are marked in green at the y-axis and pathological levels are marked in red. Notice the hippocampal sclerosis in the Lgi-1 patient and the complete recovery from the oedema in the GABA(B)R-AE-patient without a clear sign of a hippocampal atrophy. The third patient has a AMPAR-AE. The follow up in this patient shows a severe bilateral hippocampal atrophy. Nevertheless the patient improved over years.

the modified Rankin scale and also resulted in a decrease of $\mathrm{t}$ tau in these patients. The FLAIR signal also decreased in these patients (see Figure 3). In the other 8 patients without elevated t-tau levels immunosuppressive therapy had no effect on t-tau levels. A concomitant tumor had no impact at all on the t-tau levels. (see Table 2).

\section{Progranulin}

The mean CSF-PGRN levels were pathologically high in the initial NMDAR-group (CSF-PGRN $=1.55 \pm 1.1 \mathrm{ng} / \mathrm{ml}$ ) reaching significance when compared to the NMDAR under treatment group (Mann-Whitney-U test $Z=-2.5$ and $p=0.012$ ) and also when compared to the age-matched healthy group (MannWhitney-U test $Z=-2.689$ and $p=0.007$ ). Serum PGRN levels were inside normal ranges in every $\mathrm{AE}$ patient and did not change after immunosuppression (see Figure S1). CSF-PGRN and CSF-Serum ratios ranged from 0.01 to 0.1 , still CSF-PGRN is show to originate from the CNS when measured in the CSF $(26,27)$ We also could not find a correlation between SerumPGRN and CSF-PGRN in all groups (Spearman-rho coefficient $r=0.17, p=0.3$ ) (Figure $\mathbf{S} 1$ in the Supplement).After initiating the immunosuppressive therapy CSF-PGRN dropped to normal levels $(C S F-P G R N=0.75 \pm 0.2 \mathrm{ng} / \mathrm{ml})$ in the "under treatment"group.

Mean CSF-PGRN levels were normal in the Lgi-1 "initial" group $(\mathrm{CSF}-\mathrm{PGRN}=0.71 \pm 0.11 \mathrm{ng} / \mathrm{ml})$ and in the under treatment group (CSF-PGRN $=0.72 \pm 0.12 \mathrm{ng} / \mathrm{ml}$ ) (see Figure 1). Comparing these results to the age-matched group revealed significant differences (Kruskal-Wallis test: for "initial” PGRN: $p=0.009, \mathrm{X}^{2}=9.4$, for follow-up: $p=0.04, \mathrm{X}^{2}=6.3$ ). There were significantly lower levels in tamhane post-hoc in the Patients with AE (Lgi-1 initial vs. control $p=0.009$ and $p=0.041$ for the under treatment vs. control) The CSF-PGRN levels in both Caspr2 groups were inside the normal range (mean 
CSF-PRGN initially $=0.75 \pm 0.23 \mathrm{ng} / \mathrm{ml}$ and mean CSF-PGRN under treatment $=0.8 \pm 0.16 \mathrm{ng} / \mathrm{ml}$ ) without significant results when compared to the age-matched controls (Caspr2 initial vs. under treatment $p=0.35$ and Caspr 2 under treatment vs. control $p=0.92)$. There was no difference between the "initial" and the "under treatment" group in the Lgi-1 and Caspr2 cohorts, respectively.

\section{Follow Up Data}

In summary, in all cases with an elevated biomarker of neurodegeneration in the CSF a decrease of biomarkers, ab titres, and $\mathrm{mRS}$ was observed following immunosuppressive treatment in all patients (see Table $\mathbf{1}$ and Figure $\mathbf{3}$ ) regardless if the origin was postinfectious, paraneoplastic, or cryptogenic. There were two patients with all biomarkers simultaneously elevated. None of these parameters could predict a hippocampal sclerosis for sure on the one hand; on the other hand every patient who developed a sclerosis had either elevated t-tau or NfL levels with $\mathrm{t}$-tau appearing to be more predictive.

\section{DISCUSSION}

We could show for the first time that biomarkers of neurodegeneration originating from CNS are mirroring the clinical and probably neuroimmunological course of patients suffering from AE associated with antibodies to extracellular epitopes. CSF-PGRN is elevated in patients with NMDAR-AE during the acute phase. Furthermore, biomarkers of neurodegeneration such as t-tau together with CSF-NfL in Patients with AE might be predictive of the clinical outcome especially for developing a hippocampal sclerosis. The pathologically elevated biomarkers correlated with the mRS, the clinical course and the antibody titre. Besides Progranulin in acute NMDAR-AE, NfL, PGRN and t-tau did not seem to be restricted to one special autoantibody mediated AE. This may be due to the fact that the neuronal and axonal damages in general are mirrored and not the distinct pathomechanisms of each putative pathological autoantibody.

\section{Neurofilament Light Chain}

$\mathrm{NfL}$ has been proven as an excellent marker of axonal loss (28). It seems very unlikely and there has been no data on whether peripheral tumors such as teratomas nor other neuroendocrine tumors can influence the CSF-NfL levels as possible confounders in our study. The co-occurrence of MRI changes, hippocampal sclerosis, and elevated NfL levels in our Magdeburg cohort is pointing at a pathomechanism causing the edema and subsequently the FLAIR signal resulting in the axonal dysfunction and subsequently increased NfL levels in the CSF. Other than in neurodegenerative diseases such as FTD $(29,30)$ the axonal loss in AE ceases after initiating sufficient immunosuppressive therapy as seen by the group of Pranzatelli in pediatric patients with opsoclonus-myoclonus syndrome caused by antibodies with intracellular epitopes $(22,23)$. Constantinescu et al. also measured CSF-NfL in four Patients with NMDARAE with AE and one Lgi-1 patient (19). Three NMDA-patients had a status epilepticus and highly pathological CSF-NFL levels, which has been seen in SE for nearly every marker of neuronal death (20, 21). In our cohort none had a status epilepticus confounding the biomarkers. This is possibly the reason why we found normal CSF-NfL levels in all measured Patients with NMDAR-AE except for the one with postinfectious origin. The meningoencephalitis with subsequent neuronal and axonal loss before the AE might be a reason for the elevated NfL levels in this patient since the infection was only 7 weeks apart from the AE. Our results are also much more in line with the known pathomechanism in NMDAR- AE $(31,32)$ where only marginal neuronal damage occurs and the main reason for the clinical symptoms is most likely the internalization of the NMDAreceptor. The one Lgi-1 patient (without SE) in the cohort of Constantiescu et al had normal NfL levels as our entire Lgi-1 group.

Total-Tau is a better marker for neuronal death as NfL (see below). However, FLAIR intense signals in the hippocampus as a consequence of disturbances of neuronal membrane function did correlate with NfL levels in our small cohort.

\section{Total-Tau}

Total-tau is an excellent marker for neuronal death (21, 33) 4/5 patients who developed a hippocampal sclerosis had pathological elevated t-tau levels in our Magdeburg cohort $(n=13)$. This is well in line with the current concept of the pathomechanisms leading to a sclerosis (34). Although, patient 1 (see Table 2) with the Caspr2 AE who was already treated months before admission to Magdeburg had only elevated CSF-NfL and normal t-tau levels and a hippocampal sclerosis (see Table 2). Sadly, it was not possible to measure NfL and $\mathrm{t}$-tau levels in this patients initial CSF.

In sum, the measurement of t-tau may be a good marker before treatment decision in suspected autoimmune encephalitis or before deciding on the further immunosuppressive treatment but is limited to laboratories with expertise in measuring t-tau.

\section{Progranulin}

PGRN is playing a role in autoimmune mediated diseases such as rheuma or bowel disease or status epilepticus or in suppression of neuroinflammation $(30,35-37)$ Recently, EpiphrinA2 as a part of the Ephrin receptor kinase has been identified as functional receptor of PGRN and the potential of PGRN phosphorylating and activating the EpiphrinB2 receptor (38) linking it to the dysfunction in the EpiphrinB2 pathway known in AE mediated by autoantibodies against the NMDA-receptor $(39,40)$. The distribution in the fronto-temporal structures (41), the possible common link with the AE mediated by NMDAR via the EpiphrinA2-EpiphrinB2 pathway and the known role as a mediator in neuroinflammation and autoimmunity makes PGRN an interesting protein in $\mathrm{AE}$.

We detected elevated CSF-PGRN levels in our NMDApatients with a severe ongoing AE. On the other hand, CSFPGRN was low in patients suffering from Lgi-1-AE sometimes reaching levels of FTD patients (29) in contrast to Patients with NMDAR-AE and controls. The significance of this low 
CSF-PGRN is doubtful because none of the CSF-PGRN levels normalized after initiating the immunosuppressive therapy. Also most CSF-PGRN levels in the patients with Lgi-1-AE were still inside the normal range.

When looking at the t-cell or b-cell specific cytokine patterns in the patient's CSF suffering from NMDAR-AE several groups have seen a massive b-cell predominant cytokine pattern in the beginning especially in CXCL-13 levels $(42,43)$ and then a decrease in follow up. Also, cytokine pattern associated with t-cell activation were detectable throughout the course of AE without relevant changes. This course in cytokine levels could explain the elevated Progranulin levels in patients having acute NMDAR-AE.

PGRN in Serum and CSF was not elevated in patients with AE due to paraneoplastic origin although PGRN is also known as a tumor marker for certain tumors such as Lymphomas (44) The missing correlation between the serum-PGRN and CSF-PGRN is pointing at a cerebral origin of the CSF-PGRN as already seen in other diseases $(26,27)$. Overall, this result is probably due to an affection of the CSF-PGRN pathway in acute NMDAR-AE but needs more in vivo and in vitro experiments to be further examined.

One major limitation of the study is the small sample size in every subgroup tested. This fact is due to the very low numbers of patients with AE overall. Although total numbers are too small to draw a final conclusion the t-tau levels together with the CSFNFL levels seem to best characterize the stage of neuronal death in the brain. The diagnostic value of NFL levels in the CSF should be evaluated in further studies. Another limitation is that we only had follow up data in 13 patients limiting our knowledge about MRI, mRS, and ab titres. Another limitation of the study is that due to the scarcity of the diseases measurements of the biomarkers could not be done in a batch but on demand.

A larger study should be conducted to further elucidate the correlation of these interesting parameters how they could contribute to therapeutic decisions.

\section{CONCLUSION}

NfL, t-tau and PGRN could be potential biomarkers of neuronal or axonal loss in patients suffering from AE. Especially, the Patients with NMDAR-AE have elevated PGRN levels at the acute phase of the AE. This fact further strengthens the hypothesis of a pathological change in the Epiphrin receptor metabolism in NMDAR patients. CSF-PGRN may be a marker for acute NMDA-AE.

Furthermore, we strongly recommend measuring NfL and ttau in the CSF of every patient with $\mathrm{AE}$ although one biomarker

\section{REFERENCES}

1. Dalmau J, Gleichman AJ, Hughes EG, Rossi JE, Peng X, Lai MS, et al. Anti-NMDA-receptor encephalitis: case series and analysis of the effects of antibodies. Lancet Neurol. (2008) 7:1091-8. doi: 10.1016/S1474-4422(08)70224-2

2. Irani SR, Stagg CJ, Schott JM, Rosenthal CR, Schneider SA, Pettingill PR, et al. Faciobrachial dystonic seizures: the influence of immunotherapy on seizure for itself could not predict all hippocampal sclerosis in this pilot study. Pathological levels of a biomarker of neurodegeneration should be considered as an on-going $\mathrm{AE}$ and may be taken into account when planning further therapy.

\section{ETHICS STATEMENT}

This study was carried out in accordance with the recommendations of ethics committee of the University hospital Magdeburg (number 100/16). The protocol was approved by the ethics committee of the University hospital Magdeburg. All subjects gave written informed consent in accordance with the Declaration of Helsinki.

\section{AUTHOR CONTRIBUTIONS}

PK has access to all the data and takes responsibility for the data, accuracy of the data analysis, and the conduct of the research design or conceptualization of the study and analysis or interpretation of the data and drafting or revising the manuscript for intellectual content; $\mathrm{HP}$ and DB: Design and conceptualization of the study; analysis or interpretation of the data; drafting and revising the manuscript for intellectual content; LT: Conceptualization of the study; analysis of the data; drafting the manuscript for intellectual content; DV-W, JS-A, and SS: Conceptualization of the study; drafting the manuscript for intellectual content; WM, DR and FL: Conceptualization of the study; analysis and interpretation of the data; drafting and revising the manuscript for intellectual content; H-JH: Design of the study; drafting the manuscript for intellectual content.

\section{ACKNOWLEDGMENTS}

First, we have to thank our patients for the willingness to take part in this study. We have to thank Jeanette Witzke and Kerstin Kaiser at the neurochemical laboratory, Magdeburg for performing the excellent laboratory work. Furthermore, we have to thank Christian Bien at the Mara Epilepsy Center Bielefeld, Germany for laboratory work, samples and comments on the manuscript. We also appreciate the laboratory work of EUROIMMUN, Lübeck, Germany.

\section{SUPPLEMENTARY MATERIAL}

The Supplementary Material for this article can be found online at: https://www.frontiersin.org/articles/10.3389/fneur. 2018.00668/full\#supplementary-material 
antibodies: relation to antigenic specificity. J Neurol. (2014) 261:1695-705. doi: 10.1007/s00415-014-7408-6

5. van Sonderen A, Petit-Pedrol M, Dalmau J, Titulaer MJ. The value of LGI1, Caspr2 and voltage-gated potassium channel antibodies in encephalitis. Nat Rev Neurol. (2017) 13:290-301. doi: 10.1038/nrneurol. 2017.43

6. Körtvelyessy P, Bauer J, Stoppel CM, Brück W, Gerth I, Vielhaber S, et al. Complement-associated neuronal loss in a patient with CASPR2 antibodyassociated encephalitis. Neurol Neuroimmunol Neuroinflamm. (2015) 2:e75. doi: 10.1212/NXI.0000000000000075

7. Taguchi Y, Takashima S, Nukui T, Tanaka K. Reversible "brain atrophy" in anti-NMDA receptor encephalitis. Intern Med. (2011) 50:2697. doi: 10.1097/MD.0000000000006776

8. Bien CG, Vincent A, Barnett MH, Becker AJ, Blümcke I, Graus FK, et al. Immunopathology of autoantibody-associated encephalitides: clues for pathogenesis. Brain (2012) 135:1622-38. doi: 10.1093/brain/ aws082

9. Martinez-Hernandez E, Horvath J, Shiloh-Malawsky Y, Sangha N, MartinezLage M, Dalmau J. Analysis of complement and plasma cells in the brain of patients with anti-NMDAR encephalitis. Neurology (2011) 77:589-93. doi: 10.1212/WNL.0b013e318228c136

10. Sabater L, Gaig C, Gelpi E, Bataller L, Lewerenz J, Torres-Vega E, et al. A novel non-rapid-eye movement and rapid-eye-movement parasomnia with sleep breathing disorder associated with antibodies to IgLON5: a case series, characterisation of the antigen, and post-mortem study. Lancet Neurol. (2014) 13:575-86. doi: 10.1016/S1474-4422(14) 70051-1

11. Miller ZA, Rankin KP, Graff-Radford NR, Takada LT, Sturm VE, Cleveland CM, et al. TDP-43 frontotemporal lobar degeneration and autoimmune disease. J Neurol Neurosurg Psychiatry (2013) 84:956-62. doi: 10.1136/jnnp-2012-304644

12. Dahm L, Ott C, Steiner J, Stepniak B, Teegen B, Saschenbrecker SC, et al. Seroprevalence of autoantibodies against brain antigens in health and disease. Ann Neurol. (2014) 76:82-94. doi: 10.1002/ana.24189

13. Hara M, Martinez-Hernandez E, Ariño H, Armangué T, Spatola M, PetitPedrol $\mathrm{M}$, et al. Clinical and pathogenic significance of $\operatorname{IgG}$, IgA, and IgM antibodies against the NMDA receptor. Neurology (2018) 90:e1386-94. doi: 10.1212/WNL.0000000000005329

14. Schultze-Amberger J, Pehl D, Stenzel W. LGI-1-positive limbic encephalitis: a clinicopathological study. J Neurol. (2012) 259:2478-80. doi: 10.1007/s00415-012-6559-6

15. Wagner J, Witt JA, Helmstaedter C, Malter MP, Weber B, Elger CE. Automated volumetry of the mesiotemporal structures in antibody-associated limbic encephalitis. J Neurol Neurosurg Psychiatry (2014) 86:735-42. doi: 10.1136/jnnp-2014-307875

16. Wagner J, Weber B, Elger CE. Early and chronic gray matter volume changes in limbic encephalitis revealed by voxel-based morphometry. Epilepsia (2015) 56:754-61. doi: 10.1111/epi.12968

17. Finke C, Kopp UA, Prüss H, Dalmau J, Wandinger KP, Ploner CJ. Cognitive deficits following anti-NMDA receptor encephalitis. J Neurol Neurosurg Psychiatry (2012) 83:195-8. doi: 10.1136/jnnp-2011-300411

18. Leypoldt F, Gelderblom M, Schöttle D, Hoffmann S, Wandinger KP. Recovery from severe frontotemporal dysfunction at 3years after N-methyl-daspartic acid (NMDA) receptor antibody encephalitis. J Clin Neurosci. (2013) 20:611-3. doi: 10.1016/j.jocn.2012.03.036

19. Constantinescu R, Krýsl D, Bergquist F, Andrén K, Malmeström C, Asztély F, et al. Cerebrospinal fluid markers of neuronal and glial cell damage to monitor disease activity and predict long-term outcome in patients with autoimmune encephalitis. Eur J Neurol. (2016) 23:796-806. doi: 10.1111/ ene. 12942

20. Huchtemann T, Körtvélyessy P, Feistner H, Heinze HJ, Bittner D. Progranulin levels in status epilepticus as a marker of neuronal recovery and neuroprotection. Epilepsy Behav. (2015) 49:170-2. doi: 10.1016/j.yebeh.2015.06.022

21. Monti G, Tondelli M, Giovannini G, Bedin R, Nichelli PF, Trenti T, et al. Cerebrospinal fluid tau proteins in status epilepticus. Epilepsy Behav. (2015) 49:150-4. doi: 10.1016/j.yebeh.2015.04.030
22. Pranzatelli MR, Tate ED, McGee NR, Verhulst SJ. CSF neurofilament light chain is elevated in OMS (decreasing with immunotherapy) and other pediatric neuroinflammatory disorders. J Neuroimmunol. (2014) 266:75-81. doi: 10.1016/j.jneuroim.2013.11.004

23. Pranzatelli MR, McGee NR. Neuroimmunology of OMS and ANNA-1/anti-Hu paraneoplastic syndromes in a child with neuroblastoma. Neurol Neuroimmunol Neuroinflamm. (2018) 5:e433. doi: 10.1212/NXI.0000000000000433

24. Graus F, Titulaer MJ, Balu R, Benseler S, Bien CG, Cellucci T, et al. A clinical approach to diagnosis of autoimmune encephalitis. Lancet Neurol. (2016) 15:391-404. doi: 10.1016/S1474-4422(15) 00401-9

25. Kuhle J, Plattner K, Bestwick JP, Lindberg RL, Ramagopalan SV, Norgren $\mathrm{N}$, et al. A comparative study of CSF neurofilament light and heavy chain protein in MS. Mult Scler. (2013) 19:1597-603. doi: 10.1177/135245851 3482374

26. Nicholson AM, Finch NA, Thomas CS, Wojtas A, Rutherford NJ, Mielke MM, et al. Progranulin protein levels are differently regulated in plasma and CSF. Neurology (2014) 82:1871-8. doi: 10.1212/WNL.000000000 0000445

27. Wilke C, Gillardon F, Deuschle C, Dubois E, Hobert MA, Müller vom Hagen J, et al. Serum levels of progranulin do not reflect cerebrospinal fluid levels in neurodegenerative disease. Curr Alzheimer Res. (2016) 13:654-62. doi: $10.2174 / 1567205013666160314151247$

28. Steinacker P, Feneberg E, Weishaupt J, Brettschneider J, Tumani H, Andersen PM, et al. Neurofilaments in the diagnosis of motoneuron diseases: a prospective study on 455 patients. J Neurol Neurosurg Psychiatry (2016) 87:12-20. doi: 10.1136/jnnp-2015-311387

29. Körtvélyessy P, Gukasjan A, Sweeny-Reed C, Heinze HJ, Thurner L, Bittner DM. Progranulin and Amyloid- $\beta$ levels: relationship to neuropsychology in frontotemporal and Alzheimer's Disease. J Alzheimers Dis (2015) 46:375-80. doi: 10.3233/JAD-150069

30. Kortvelyessy P, Heinze HJ, Prudlo J, Bittner D. CSF biomarkers of neurodegeneration in progressive non-fluent aphasia and other forms of frontotemporal dementia: clues for pathomechanisms? Front Neurol. (2018) 9:504. doi: 10.3389/fneur.2018.00504

31. Planagumà J, Leypoldt F, Mannara F, Gutiérrez-Cuesta J, MartínGarcía E, Aguilar E, et al. Human N-methyl D-aspartate receptor antibodies alter memory and behaviour in mice. Brain (2015) 138:94-109. doi: 10.1093/brain/awu310

32. Kreye J, Wenke NK, Chayka M, Leubner J, Murugan R, Maier NB, et al. Human cerebrospinal fluid monoclonal N-methyl-D-aspartate receptor autoantibodies are sufficient for encephalitis pathogenesis. Brain (2016) 139:2641-52. doi: 10.1093/brain/aww208

33. Spillantini MG, Goedert M. Tau pathology and neurodegeneration. Lancet Neurol. (2013) 12:609-22. doi: 10.1016/S1474-4422(13) 70090-5

34. Thom M, Eriksson S, Martinian L, Caboclo LO, McEvoy AW, Duncan JS, et al. Temporal lobe sclerosis associated with hippocampal sclerosis in temporal lobe epilepsy: neuropathological features. J Neuropathol Exp Neurol. (2009) 68:928-38. doi: 10.1097/NEN.0b013e3181b05d67

35. Cenik B, Sephton CF, Kutluk Cenik B, Herz J, Yu G. Progranulin: a proteolytically processed protein at the crossroads of inflammation and neurodegeneration. J Biol Chem. (2012) 287:32298-306. doi: 10.1074/jbc.R112.399170

36. De Muynck L, Van Damme P. Cellular effects of progranulin in health and disease. J Mol Neurosci. (2011) 45:549-60. doi: 10.1007/s12031-011-9553-Z

37. Jian J, Li G, Hettinghouse A, Liu C. Progranulin: A key player in autoimmune diseases. Cytokine (2016) 101:48-55. doi: 10.1016/j.cyto.2016. 08.007

38. Neill T, Buraschi S, Goyal A, Sharpe C, Natkanski E, Schaefer L, et al. EphA2 is a functional receptor for the growth factor progranulin. J Cell Biol. (2016) 215:687-703. doi: $10.1083 /$ jcb.201603079

39. Mikasova L, De Rossi P, Bouchet D, Georges F, Rogemond V, Didelot A, et al. Disrupted surface cross-talk between NMDA and Ephrin-B2 receptors in anti-NMDA encephalitis. Brain (2012) 135:1606-21. doi: 10.1093/brain/ aws092 
40. Planagumà J, Haselmann H, Mannara F, Petit-Pedrol M, Grünewald B, Aguilar E, et al. Ephrin-B2 prevents N-methyl-D-aspartate receptor antibody effects on memory and neuroplasticity. Ann Neurol. (2016) 80:388-400. doi: 10.1002/ana.24721

41. Daniel R, He Z, Carmichael KP, Halper J, and Bateman A. Cellular localization of gene expression for progranulin. $J$ Histochem Cytochem. (2000) 48:999-1009. doi: 10.1177/00221554000 4800713

42. Liba Z, Kayserova J, Elisak M, Marusic P, Nohejlova H, Hanzalova J, et al. Anti$\mathrm{N}$-methyl-D-aspartate receptor encephalitis: the clinical course in light of the chemokine and cytokine levels in cerebrospinal fluid. J Neuroinflammation (2016) 13:55. doi: 10.1186/s12974-016-0507-9

43. Leypoldt F, Höftberger R, Titulaer MJ, Armangue T, Gresa-Arribas $\mathrm{N}$, Jahn $\mathrm{H}$, et al. Investigations on CXCL13 in Anti-N-Methyl-Daspartate receptor encephalitis: a potential biomarker of treatment response. JAMA Neurol (2014) 72:180-6. doi: 10.1001/jamaneurol.201 4.2956

44. Arechavaleta-Velasco F, Perez-Juarez CE, Gerton GL, Diaz-Cueto L. Progranulin and its biological effects in cancer. Med Oncol. (2017) 34:194. doi: $10.1007 /$ s12032-017-1054-7
Conflict of Interest Statement: PK has received consulting fees from Eisai (Germany). JS-A obtained honoraria for speaking engagements from Boehringer Ingelheim (Germany) and Bristol-Myers Squibb (Germany). LT: Saarland University, LT and others filed 61/730,772 which covers means and methods for detecting autoimmune disorders in which progranulin antibodies may be involved. FL runs an antibody detection laboratory in Kiel, Germany where part of the work has been performed.

The remaining authors declare that the research was conducted in the absence of any commercial or financial relationships that could be construed as a potential conflict of interest.

Copyright () 2018 Körtvelyessy, Prüss, Thurner, Maetzler, Vittore-Welliong, Schultze-Amberger, Heinze, Reinhold, Leypoldt, Schreiber and Bittner. This is an open-access article distributed under the terms of the Creative Commons Attribution License (CC BY). The use, distribution or reproduction in other forums is permitted, provided the original author(s) and the copyright owner(s) are credited and that the original publication in this journal is cited, in accordance with accepted academic practice. No use, distribution or reproduction is permitted which does not comply with these terms. 